\title{
Quirky composite dark matter
}

\author{
Graham D. Kribs, ${ }^{1}$ Tuhin S. Roy, ${ }^{1}$ John Terning, ${ }^{2}$ and Kathryn M. Zurek ${ }^{3,4}$ \\ ${ }^{1}$ Department of Physics, University of Oregon, Eugene, Oregon 97403, USA \\ ${ }^{2}$ Department of Physics, University of California, Davis, California 95616, USA \\ ${ }^{3}$ Particle Astrophysics Center, Fermi National Accelerator Laboratory, Batavia, Illinois 60510, USA \\ ${ }^{4}$ Department of Physics, University of Michigan, Ann Arbor, Michigan 48109, USA
}

(Received 9 December 2009; published 3 May 2010)

\begin{abstract}
We propose a new dark matter candidate, "quirky dark matter," that is a scalar baryonic bound state of a new non-Abelian force that becomes strong below the electroweak scale. The bound state is made of chiral quirks: new fermions that transform under both the new strong force as well as in a chiral representation of the electroweak group, acquiring mass from the Higgs mechanism. Electric charge neutrality of the lightest baryon requires approximately degenerate quirk masses which also causes the charge radius of the bound state to be negligible. The abundance is determined by an asymmetry that is linked to the baryon and lepton numbers of the universe through electroweak sphalerons. Dark matter elastic scattering with nuclei proceeds through Higgs exchange as well as an electromagnetic polarizability operator which is just now being tested in direct detection experiments. A novel method to search for quirky dark matter is to look for a gamma-ray "dark line" spectroscopic feature in galaxy clusters that result from the quirky Lyman-alpha or quirky hyperfine transitions. Colliders are expected to dominantly produce quirky mesons, not quirky baryons, consequently large missing energy is not the primary collider signal of the physics associated with quirky dark matter.
\end{abstract}

DOI: 10.1103/PhysRevD.81.095001

PACS numbers: 95.35.+d, 12.60.-i

\section{INTRODUCTION}

Dark matter is a big mystery. Weakly interacting massive particles (WIMPs) provide one interesting class of particles to serve as dark matter. There are, nevertheless, two main puzzles with typical WIMP candidates:

(1) Abundance is determined by thermal freeze-out. While a thermal freeze-out origin can yield the observed cosmological abundance when the annihilation cross section is tuned to roughly $1 \mathrm{pb}$, this mechanism is entirely unrelated to the origin of matter, which arises from an asymmetry. The observational relation between the dark matter density and the baryonic density, $\rho_{D} \simeq 5 \rho_{B}$, is a coincidence.

(2) Elementary WIMPs with electroweak interaction strength couplings to standard model (SM) fermions generically have very strong constraints from direct detection bounds. To be "safe," WIMP interactions with the SM must be subweak strength, and typically, their mass determined by a mechanism unrelated to electroweak symmetry breaking.

We propose a new model of dark matter that tackles both problems. The first problem can be addressed if the dark matter abundance is linked to the baryon abundance. This has been considered before, for example, in the context of technibaryon dark matter [1-6]. In these models, electroweak sphalerons reprocess baryon and lepton asymmetries into technibaryon asymmetry. The constituents are electroweak charged, while the technibaryon composite dark matter is neutral. The sphalerons generate a relation between the number densities of leptons, baryons, and tech- nibaryons,

$$
n_{\ell}-n_{\bar{\ell}} \sim n_{b}-n_{\bar{b}} \sim n_{t b}-n_{\overline{t b}},
$$

where $n_{\ell}-n_{\bar{\ell}}, n_{b}-n_{\bar{b}}$, and $n_{t b}-n_{\bar{t} \bar{b}}$ represent the lepton, baryon, and technibaryon asymmetries, and the exact proportions are $\mathcal{O}(1)$ depending on the electroweak charges of the technibaryon constituents. Cosmologically $\rho_{D} / \rho_{B} \approx 5$, so that the relation Eq. (1.1) implies the dark matter mass $M \sim 5 \mathrm{GeV}$. If this were the end of the story, technibaryon dark matter (or any other weak scale model which connects the dark matter to the baryon asymmetry, e.g. $[7,8]$ ) would be ruled out. However, if the dark matter constituents are just becoming nonrelativistic as the sphalerons are decoupling, there is an exponential Boltzmann suppression in the technibaryon asymmetry relative to the lepton and baryon asymmetries, implying the dark matter can naturally have an electroweak scale mass. The other possible solution to the dark matter baryon coincidence places the GeV scale dark matter in a hidden sector weakly coupled to the SM sector [9-17].

The second problem can, in fact, be ingeniously solved by compositeness. In technicolor theories, technifermions interact with the SM through renormalizable interactions, while a composite technibaryon dark matter candidate is charge and electroweak neutral. This eliminates renormalizable interactions with the SM below the electroweak scale, leaving only higher dimensional operators [3,4]. In the early 1990s this was thought to be an unfortunate result-dark matter could not be observed in the thenforeseeable future. Given the direct detection bounds today (e.g. $[18,19])$, this becomes a "feature" of our composite 
dark matter model that we exploit to naturally suppress the direct detection cross sections.

The residual electroweak-mediated direct detection cross section of composite dark matter arises from "form factor" suppression, somewhat analogous to the suppression of the photon coupling to neutrons at energies much smaller than $\Lambda_{\mathrm{QCD}}$. For example, the leading order operators that couple scalar technibaryon dark matter to the SM arise at dimension-6 (charge radius) [4] and dimension-7 (chromomagnetic polarizability) [3], suppressed by two or three powers of $\Lambda_{\mathrm{TC}}$.

What was not fully appreciated in the 1990s is that both of these operators can be eliminated. The charge radius vanishes in a limit in which the current masses of the constituents are equal. The chromomagnetic polarizability vanishes when the constituents do not carry ordinary QCD color. While this suggests rethinking technicolor dark matter (e.g. $[5,6,20])$, the model building difficulties of realizing a fully successful technicolor model incorporating flavor as well as avoiding electroweak precision constraints remains daunting.

In this paper, we take a different approach, in the spirit of the Hidden Valley [21] and Kang and Luty's quirks [22]. We retain the new strong dynamics, but discard their role in electroweak symmetry breaking. The new strong dynamics gets strong at a scale below the electroweak scale. We call the candidate that arises in this approach quirky dark matter (QDM). The simplest quirky dark matter model, as we will describe, contains exactly the same gauge and matter content as that of minimal $S U(2)$ technicolor with two flavors. Amusingly, what was originally a problem of minimal technicolor - namely the possibility that the vacuum aligned to an electroweak-preserving state [23] - is now a feature here since we utilize the ordinary Higgs mechanism to break electroweak symmetry. Indeed, we do not want the strong dynamics to break electroweak symmetry (even a little bit) lest we run into electroweak precision constraints.

With an ordinary Higgs present, quirks can obtain their mass through the Higgs mechanism just like quarks and leptons. This has several implications: New contributions to the electroweak oblique corrections arise; we estimate them below. Quirky dark matter can interact with nuclei of direct detection experiments through Higgs exchange; this leads to an ordinary elastic scattering cross section that is right near the current bounds for a light Higgs boson. Finally, assuming the new strong force confines, new operators involving the Higgs are present that can allow the new glueballs to decay, independent of the quirk mass.

\section{MODEL}

\section{A. Field content}

The model of quirky dark matter that we mainly wish to consider consists of two flavors of quirks transforming under a new strongly interacting sector, $S U(2)_{Q}$, that here-
TABLE I. Particle content and charges under the gauge and global symmetries.

\begin{tabular}{lcccc}
\hline \hline & $S U(2)_{Q}$ & $S U(2)_{L}$ & $U(1)_{Y}$ & $U(1)_{\mathrm{QB}}$ \\
\hline$\xi_{Q}=\left(\xi_{U}, \xi_{D}\right)$ & $\mathbf{2}$ & $\mathbf{2}$ & 0 & $+1 / 2$ \\
$\xi_{\bar{U}}$ & $\mathbf{2}$ & $\mathbf{1}$ & $-1 / 2$ & $-1 / 2$ \\
$\xi_{\bar{D}}$ & $\mathbf{2}$ & $\mathbf{1}$ & $+1 / 2$ & $-1 / 2$ \\
\hline \hline
\end{tabular}

after we call "quirkcolor." Variations of this, with different numbers of quirk flavors and quirkcolors are also possible; we will remark on the possibility of more quirk flavors later in the paper. We assume that the quirkcolor coupling constant gets strong below the weak scale. The particle content and charges under the gauge and global symmetries are given in Table I in terms of two-component spinors. This assignment is chiral under the electroweak group, and thus requires Yukawa interactions with the Higgs,

$$
\mathcal{L}_{Y}=\lambda_{U} \xi_{Q} H \xi_{\bar{U}}+\lambda_{D} \xi_{Q} H^{\dagger} \xi_{\bar{D}}
$$

to give current masses to the quirks, $m_{q}=\lambda_{q} v$, for $q=$ $U, D$. We enforce a global $U(1)_{\mathrm{QB}}$ "quirky baryon number" that forbids the mass terms $\xi_{Q} \xi_{Q}$ and $\xi_{\bar{U}} \xi_{\bar{D}}$ and ensures our quirky dark matter candidate is stable (on at least cosmological time scales).

Since QDM contains additional matter that acquires mass exclusively through the Higgs mechanism, there are new contributions to the electroweak precision parameters. The quirks in our model are weakly coupled at the scale of their mass, and so we can perturbatively calculate $\Delta S, \Delta T$ [24]. Generically, $\Delta S=1 /(3 \pi) \simeq 0.1$, while $\Delta T$ depends on the splitting within the quirky electroweak doublets. As we will show below, eliminating the charge radius operator requires negligible splitting between the current masses of the quirks. As a consequence, the contribution to $T$ from this sector is negligible. The minimal model therefore has $\Delta S \simeq 0.1, \Delta T \simeq 0.0$, which is roughly at the 95\% C.L. contour when comparing against LEP electroweak working group fits $[25,26]$. Suffice to say it is a straightforward (but unenlightening) exercise to slightly extend the model to give a additional contributions to $T$ (and $S$ ) that result in a model fully consistent with electroweak precision data.

In the minimal model, with the only particles transforming under $S U(2)_{Q}$ given by that in Table I, the quirkcolor group confines. The global $S U(4) \sim S O(6)$ symmetry is broken to $S O(5)$ and we have $15-10=5$ pseudoNambu-Goldstone bosons. The large current quirk masses ensure these composites are massive, forming "quirkonia" bound states with a spectrum similar to heavy quarkonia [27]. We assume the quirkonia masses are sufficiently heavy to avoid LEP II bounds (i.e., quirks heavier than about $100 \mathrm{GeV}$ ). Existing Tevatron bounds, as well as prospects for future detection at Tevatron and LHC, will be studied in a future paper. 
Confinement of $S U(2)_{Q}$ leads to quirkcolor glueballs. These glueballs decay through higher dimensional operators into SM matter. Depending on the scales, however, their lifetime may be very long [22,28], potentially leading to cosmological problems depending on the quirk masses and the confinement scale. In QDM, there are additional operators due to interactions with the Higgs. These interactions are written with an estimate of their contribution to the glueball width in Sec. VI.

Our quirkcolor group $S U(2)_{Q}$, however, does not necessarily need to confine, if additional (massless) flavors transforming only under $S U(2)_{Q}$ are present. This provides an interesting possibility in which quirkcolor flows to a conformal field theory without confinement. We emphasize that, for much of our discussion below, essentially none of our calculations depend on the scale (or existence) of confinement, so long as it is sufficiently smaller than the inverse Bohr radius of the bound states so that reliable nonrelativistic calculations can be performed. To this end, we need the quirkcolor coupling, evaluated at the scale of the inverse Bohr radius, to be perturbative. The situation is quite analogous to heavy quarkonia. Indeed, we employ much of the formalism of nonrelativistic effective theories developed for quarkonia and apply it directly to QDM. The systematic derivation of the nonrelativistic limit from the relativistic degrees of freedom, following the quarkonia literature, is outlined in Appendix A.

Our composites include "mesons" and "baryons" depending on whether or not they carry the nonzero $U(1)_{\mathrm{QB}}$ quirky baryon number. To satisfy the Pauli exclusion principle, the wave function of baryons must be antisymmetrized with respect to all quantum numbers leading to a detailed spectrum of allowed states. Details outlining the construction of the bound state spectrum from the relativistic spinors to the nonrelativistic mesons and baryons are given in Appendix A. Here we simply quote the results and present the baryon spectrum that is relevant for dark matter and its interactions, given in Fig. 1.

The dynamics and binding energies of the quirky baryons is our primary interest in this paper. For this, we need to construct the nonrelativistic potential, $V_{s}(r)$. Formally, this is possible in the limit $m_{q} \gg m_{q} v \gg m_{q} v^{2}$, where the potential is dominated by single quirkcolor gauge field exchange between the massive quirks. ${ }^{1}$ Since our binding quirkcolor force is non-Abelian, the strength of the potential depends on the representation of the constituents. For our model, the quirks are in the fundamental representation, which gives a potential

\footnotetext{
${ }^{1}$ If $S U(2)_{Q}$ confines at a scale $\Lambda_{Q}$, we actually need $m_{q} v \gg$ $m_{q} v^{2} \gtrsim \Lambda_{Q}$. This is because in the alternate limit $m_{q} v \gg$ $\Lambda_{Q} \gg m_{q} v^{2}$ one must integrate out the physical scale $\Lambda_{Q}$ before the potential can be properly defined. This procedure leads to an additional nonperturbative part in the potential that contains nonlocal quirkcolor gauge field correlators.
}
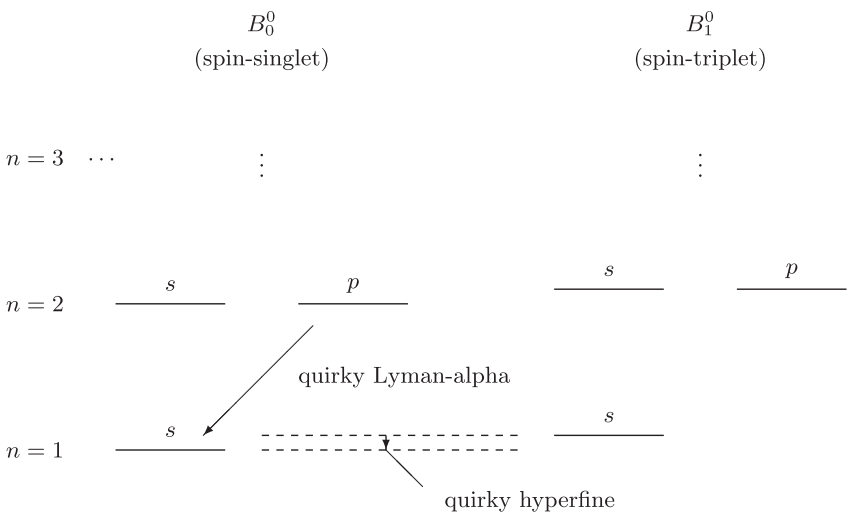

FIG. 1. Sketch of the quantum mechanical energy spectrum of our quirky dark matter composite with the ground state and several excited states shown. Our notation $B_{S}^{q}$ corresponds to baryonic states with total electric charge $q$ and total spin $S$. We have included $\mathcal{O}\left(\bar{\alpha}^{2}\right)$ (quirky Lyman-alpha) and $\mathcal{O}\left(\bar{\alpha}^{4}\right)$ (quirky hyperfine) splittings, but do not show the $\mathcal{O}\left(\bar{\alpha}^{5}\right)$ (quirky Lamb shift) splittings or other higher-order effects. The lightest electrically charged baryons $B_{1}^{ \pm}$(not shown), have spin one, and are slightly heavier than $B_{1}^{0}$ due to subdominant electromagnetic corrections to the potential.

$$
V_{s}(r)=-\frac{\bar{\alpha}(r)}{r}
$$

where $^{2} \bar{\alpha}(r) \equiv C_{2}(\mathbf{2}) \alpha_{Q}(r)=\frac{3}{4} \alpha_{Q}(r)$, and $\alpha_{Q}(r)$ is the strength of the quirkcolor force evaluated at the scale $1 / r$. This potential strictly arises from the one quirkcolor gluon exchange in the singlet channel, $\left(\sum_{a} t_{i j}^{a} t_{k l}^{a}\right) \delta_{j l}=$ $C_{2}(\mathbf{2}) \delta_{i j}$. This potential is similar to the Coulombic potential used to determine the bound states of the hydrogen atom. However, the non-Abelian nature of the quirkcolor binding force implies $\alpha_{Q}(r)$ is scale dependent, which to leading $\log$ is given by the $\beta$ function $[29,30]$

$$
\alpha_{Q}(r)=\alpha_{Q}\left(r_{B}\right)\left(1+\frac{\alpha_{Q}\left(r_{B}\right)}{3 \pi}\left(11-N_{f}\right) \ln \left(r / r_{B}\right)\right)
$$

Here $r_{B} \equiv\left[\bar{\alpha}\left(r_{B}\right) \mu\right]^{-1}$ is the analogue of the Bohr radius in the hydrogen atom and gives the typical size of the bound states. We have written the $r$-dependent correction to the potential for a general number of quirkcolor flavors for completeness. The model on which we concentrate our attention has $N_{f}=2$, as defined before in Table I.

There is an additional correction to the potential, Eq. (2.2), which arises from Higgs exchange between the quirks. This contribution is exponentially suppressed due to the massive Higgs propagator; however, it is interesting to estimate the size of the additional contribution to the bound state potential,

\footnotetext{
${ }^{2}$ We drop the subscript $Q$ when writing $\bar{\alpha}$ to keep the notation as simple as possible.
} 


$$
\Delta V(r) \sim-\frac{\lambda_{q}^{2}}{4 \pi r} \exp \left[-m_{h} r\right]
$$

For at least a semiperturbative analysis to hold, $\lambda_{q}^{2} / 4 \pi \lessgtr$ 0.5 which implies $m_{q} \lesssim 450 \mathrm{GeV}$ and thus $M \lessgtr$ $900 \mathrm{GeV}$. The size of the exponential suppression depends on $m_{h}$ and $r$, where the characteristic size relevant to the bound state is the Bohr radius $r=r_{B}$. Suffice to say, we need $m_{h}>r_{B}^{-1}$ to ensure Higgs exchange is negligible. As we will see, the lower bound on $r_{B}^{-1}$ from electromagnetic polarizability is in the tens of $\mathrm{GeV}$ range, so for a significant range of parameter space, we find that Higgs exchange even with Higgs mass at the LEP 2 bound is negligible. To the extent that we consider at all the semiperturbative regions where $\bar{\alpha}$ and $M$ are simultaneously large, we assume the Higgs mass is large enough that we can neglect Higgs exchange in the bound state calculation.

\section{B. Binding energies and splittings}

Our quirkcolor singlet bound states can be described by a Schrödinger-like equation with a quirkcolor force potential given by Eqs. (2.2) and (2.3). In the limit that the $\log r$ piece can be neglected, the potential becomes purely Coulombic - the same as the hydrogen atom-with the replacements $m_{e} \leftrightarrow \mu$ and $\alpha_{e m} \leftrightarrow \bar{\alpha}$. One can formally approach the Coulombic limit if enough additional flavors are present to lead to a nearly scale-invariant quirkcolor theory while $\bar{\alpha}$ remains perturbative.

We will be interested in the regime where $\bar{\alpha}$ is perturbative but not necessarily small, and with exactly two flavors of quirks as given in Table I. Hence, our nonrelativistic potential has unavoidable $\log r$ dependence. In our calculations below, we express the effect of the log term as coefficients that multiply the exact solutions obtained in the case of a purely Coulombic potential. The coefficients have been computed by numerically solving the Schrödinger equation including the log term for a few specific choices of $\bar{\alpha}$.

The binding energies of the $n$th excited state of the quirkcolor singlets is given by

$$
E_{n}=-k_{n} \frac{\bar{\alpha}^{2} \mu}{2 n^{2}}
$$

expressed in terms of the reduced mass of the quirks, $1 / \mu \equiv 1 / m_{U}+1 / m_{D}$. The $n$-dependent constant $k_{n}$ captures the difference between a pure Coulombic potential and our non-Abelian quirkcolor theory. Using our numerical evaluation of the Schrödinger equation, we find the energy levels of the first two states are corrected by $k_{1} \simeq(1.2,1.3,1.4)$ and $k_{2} \simeq(1.9,2.3,2.8)$ for $\bar{\alpha}\left(r_{B}\right)=$ $(0.2,0.3,0.4)$ and $N_{f}=2$.

Next, we consider the hyperfine structure of the quirkcolor singlet bound states. The spin of each of the constituent quirks generates a dipole quirkcolor "magnetic" field which leads to a spin-spin interaction,

$$
H_{h f}=\frac{8 \pi}{3} \frac{\bar{\alpha}}{m_{U} m_{D}} \vec{S}_{1} \cdot \vec{S}_{2} \delta^{3}(\vec{r})+\cdots,
$$

where the terms we have neglected do not contribute to the angular momentum $\ell=0$ states. This is the leading nonrelativistic contribution to the hyperfine structure. (Relativistic corrections, such as Thomas precession [31], are small so long as the quirk masses are much larger than the strong scale.) Sandwiching this Hamiltonian between states of the unperturbed potential gives a splitting proportional to the (unperturbed) wave function at the origin,

$$
\left|\psi_{1,0}(0)\right|^{2}=c_{1,0} \frac{\left[\mu \bar{\alpha}\left(r_{B}\right)\right]^{3}}{\pi} .
$$

This is the familiar result from the hydrogen atom, except for the constant $c_{n, \ell}$ which differs from one due to the $\log r$ term in our nonrelativistic potential. Numerically calculating the coefficient for $(n, \ell)=(1,0)$, we find $c_{1,0} \simeq$ $(0.5,0.4,0.3)$ for $\bar{\alpha}\left(r_{B}\right)=(0.2,0.3,0.4)$ and $N_{f}=2$. The hyperfine splitting is thus estimated from Eq. (2.7) to be

$$
E_{h f}=c_{1,0} \frac{\mu^{3} \bar{\alpha}^{4}}{3 m_{U} m_{D}} \begin{cases}2 & \text { spin triplet } \\ -6 & \text { spin singlet. }\end{cases}
$$

As long as the quirk masses satisfy $\left|m_{U}-m_{D}\right|<E_{h f}$, the electrically neutral spin-singlet baryon $B_{0}^{0}$ is lighter than the electrically charged $q=(+1,0,-1)$ spin-triplet baryons $B_{1}^{q}$, in agreement with [1]. This requires our quirk current masses to be very nearly degenerate, $m_{U} \simeq m_{D}$. Hereafter, we use $B_{0}^{0}$ to denote our quirky dark matter candidate in its ground state, $n=1$. We illustrate the spectrum of the ground and first excited baryonic states in Fig. 1.

\section{QUIRKY DARK MATTER ABUNDANCE}

Stable quirks transforming under a chiral representation of the electroweak group have an abundance that is necessarily related to the baryon and lepton abundance through the electroweak phase transition. That such a relationship is inevitable was discovered in early work on the technibaryon abundance from technicolor theories [2]. There it was shown that baryons and technibaryons could have a common origin, since sphalerons intermix baryon, lepton, and technibaryon numbers. More interestingly, Ref. [2] showed that the large mass of the technibaryons causes an additional Boltzmann suppression of their abundance, roughly scaling as $\exp \left[-M_{*} / T_{*}\right]$, where $M_{*}$ is the mass of the technibaryon at the critical temperature $T_{*}$ where sphalerons shut off. This allows for $\mathrm{TeV}$ mass technibaryons to nevertheless yield roughly the right dark matter abundance today (for recent calculations in technicolor theories, see e.g. [5]). There are three global flavor quantum numbersbaryon, lepton, and technibaryon number-while the sphaleron violates only one linear combination, leaving two anomaly-free invariants [7]. Hence, while baryon and technibaryon numbers are related to one another, one cannot 
determine technibaryon number uniquely from just baryon number. Instead, baryon, lepton, and technibaryon numbers are ultimately determined in terms of linear combinations of two invariants which can be taken to be $B-L$ number [32] and another combination involving both baryon (or lepton) number and technibaryon number [7].

The abundance of quirky dark matter is determined by an analysis similar to that of technibaryon dark matter. The main difference between our calculation below and that of [2] is that quirkcolor is assumed to be weakly coupled through the electroweak phase transition. Sphalerons therefore yield an (asymmetric) abundance of quirks instead of quirky baryons. To also exploit the Boltzmann suppression of quirky baryon number, quirks must acquire mass before sphalerons shut off, which can occur if the electroweak phase transition is not first order. The Boltzmann suppression for the abundance of quirks is therefore proportional to $\exp \left[-\lambda_{q} v\left(T_{*}\right) / T_{*}\right]$, where $v\left(T_{*}\right)$ is the electroweak vev at the critical temperature $T_{*}$. The final ratio of quirky dark matter abundance to baryon abundance is determined by three quantities: the two primordial anomaly-free $U(1)$ invariants and the ratio $m_{q}\left(T_{*}\right) / T_{*}=\lambda_{q} v\left(T_{*}\right) / T_{*}$. In principle, $v\left(T_{*}\right)$ and $T_{*}$ can be calculated within our theory. This requires incorporating the effects of quirks on the phase transition. ${ }^{3}$

Following the classic calculation of [34], the divergence of the baryon, lepton, and quirky baryon currents can be constructed from

$$
\partial_{\mu} j^{\mu}=\frac{N g^{2}}{64 \pi^{2}} \epsilon^{\mu \nu \rho \sigma} F_{\mu \nu}^{a} F_{\rho \sigma}^{a}
$$

where only $S U(2)_{W}$ effects on $N$ electroweak doublets are considered. It is straightforward to determine that the sphaleron carries $B=N_{g} / 2, L=N_{g} / 2$, and $D=N_{D} / 2$ charge, where $N_{g}=3$ is the number of SM generations and $N_{D}=1$ is the number of electroweak doublets that carry quirky baryon charge. We normalize the quirks to carry $1 / N_{Q}=1 / 2$ quirky baryon charge, given $N_{Q}=2$ quirkcolors, precisely analogous to the $1 / N_{c}=1 / 3$ baryon number normalization of quarks.

This result implies sphalerons violate the global $U(1)$ number $B+L+\frac{N_{D}}{N_{g}} D$. The orthogonal combinations that are preserved can be taken to be $I_{1} \equiv B-L$ and $I_{2} \equiv B-$ $\frac{N_{g}}{N_{D}} D$ (or $L-\frac{N_{g}}{N_{D}} D$ ) [7]. Using the standard techniques [32,35], we enforce the following relations among the chemical potentials: (i) electric neutrality, and (ii) the van-

\footnotetext{
${ }^{3}$ Examples of theories with additional chiral fermions with large Yukawa couplings have been considered, e.g., [33]. There it was found that the electroweak phase transition was weakened (without superpartners), which is not inconsistent with our expectations, though we leave a more detailed analysis to future work.
}

ishing of the chemical potential of the Higgs boson. With these conditions, and taking $N_{g}=3$ and $N_{D}=1$, we obtain

$$
\begin{gathered}
B=\frac{\left[36 f(x)+4 f(x)^{2}\right] I_{1}+[17+2 f(x)] I_{2}}{17+113 f(x)+13 f(x)^{2}} \\
L=\frac{-\left[17+77 f(x)+9 f(x)^{2}\right] I_{1}+[17+2 f(x)] I_{2}}{17+113 f(x)+13 f(x)^{2}} \\
D=f(x) \frac{[36+4 f(x)] I_{1}-[111+13 f(x)] I_{2}}{51+339 f(x)+39 f(x)^{2}},
\end{gathered}
$$

where

$$
f(x)=\frac{3}{2 \pi^{2}} \int_{0}^{\infty} \frac{y^{2}}{\cosh ^{2} \frac{1}{2} \sqrt{y^{2}+x^{2}}}
$$

in terms of $x=m_{q}\left(T_{*}\right) / T_{*}$. The mass density ratio is, therefore,

$$
\begin{aligned}
\frac{\rho_{D}}{\rho_{B}} & =\frac{D}{B} \frac{M}{m_{p}} \\
& =f(x) \frac{[36+4 f(x)] I_{1}-[111+13 f(x)] I_{2}}{6 f(x)[18+2 f(x)] I_{1}+[51+6 f(x)] I_{2}} \frac{M}{m_{p}},
\end{aligned}
$$

where $M$ is the mass of $B_{0}^{0}$ in its ground state. In Fig. 2 we show contours of the resulting quirky dark matter density $\rho_{D} / \rho_{B}$ within the parameter space of the two primordial invariants $I_{1}$ and $I_{2}$. Pure leptogenesis, which corresponds to $I_{1}=-L$ and $I_{2}=0$, immediately implies $D=B / 3$, independent of $f(x)$. This mechanism is not viable since the mass of quirky dark matter would have to be $M \simeq$ $5 m_{p} B / D \simeq 15 \mathrm{GeV}$, which is ruled out by direct collider searches. Pure baryogenesis or some mixture of all three remains perfectly viable so long as the lepton number of our universe remains unknown.

\section{PROSPECTS FOR DIRECT DETECTION}

\section{A. Overview}

There are three basic ways that quirky dark matter could potentially be found in direct detection experiments:

(i) Elastic scattering through Higgs exchange.

(ii) Elastic scattering through higher dimensional operators.

(iii) Inelastic scattering through an excited quirky baryonic state.

The third way, inelastic scattering, has been considered before in general [36] and recently in the context of composite inelastic dark matter [37,38]. Quirky dark matter is more akin to Ref. [36], where it was shown that one needs 

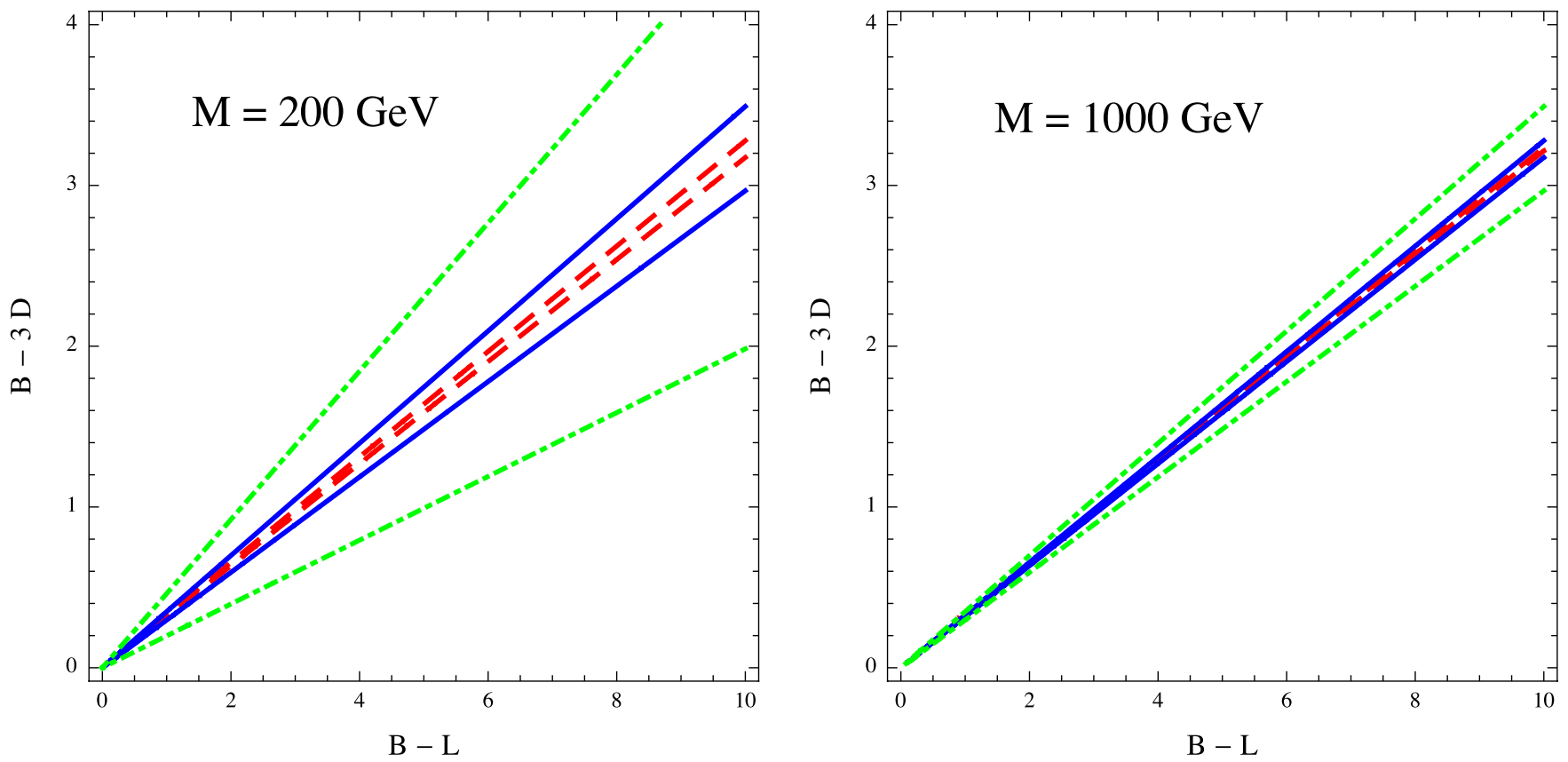

FIG. 2 (color online). Contour plots of the density ratio $\rho_{D} / \rho_{B}=(1,5,25)$ shown by dashed, solid, and dot-dashed (red, blue, and green) lines. The axes are the invariants $\left(I_{1}, I_{2}\right) \equiv(B-L, B-3 D)$ in arbitrary units; a mirror symmetric plot can be obtained taking $\left(I_{1}, I_{2}\right) \rightarrow\left(-I_{1},-I_{2}\right)$. The plot on the left has $M=200 \mathrm{GeV}, x=0.25$, and on the right $M=1000 \mathrm{GeV}, x=0.25$. The plots demonstrate that a viable region exists with $\rho_{D} / \rho_{B} \simeq 5$, corresponding to a "bathtub ring" around a valley in $\left(I_{1}, I_{2}\right)$ space. The bottom of the valley has $\rho_{D} / \rho_{B} \simeq 0$.

fairly small splittings, up to about $10 \mathrm{MeV}$, to allow for inelastic recombination. The smallest splitting in quirky dark matter is the hyperfine splitting. Combining a rough bound from LEP II, $m_{q} \gtrsim 100 \mathrm{GeV}$, with $\bar{\alpha} \gtrsim 0.1$ to satisfy direct detection bounds (explicitly shown later in this section), we find the hyperfine splitting $E_{h f} \simeq$ $2 \bar{\alpha}^{4} \mu / 3 \gtrsim 30 \mathrm{MeV}$. So, we do not anticipate inelastic scattering or inelastic recombination in direct detection experiments.

\section{B. Higgs exchange}

Our quirks acquire mass through the Higgs mechanism, and hence $B_{0}^{0}$ has interactions with matter through Higgs exchange. Just as the Higgs couples to the $\bar{q} q$ content of the nucleon through $\langle N|\bar{q} q| N\rangle$, the Higgs also couples to the quirk-quirk content of our quirky baryonic dark matter $\left\langle B_{0}^{0}|q q| B_{0}^{0}\right\rangle$. Unlike the nucleon, however, the quirkcolor gluon condensate is presumed to give a negligible contribution to the quirky baryon mass. The calculation of Higgs exchange is most easily done in the low energy effective theory below the scale of the quirky baryon. Then we can treat $B_{0}^{0}$ as simply a complex scalar with a renormalizable interaction with the Higgs,

$$
\mathcal{L} \simeq M h B_{0}^{0 *} B_{0}^{0}
$$

where this interaction assumes the mass of $B_{0}^{0}$ arises mostly from the current quirk masses, i.e., $M \simeq m_{U}+m_{D}$. With this interaction, we can use the results of Refs. [39,40], which considered the scattering of real scalars through Higgs exchange, and read off the direct detection cross section. ${ }^{4}$ We obtain

$$
\sigma(\text { nucleon })=\frac{\mu(D, n)^{2}}{4 \pi A^{2} m_{h}^{4}}\left(Z f_{p}+(A-Z) f_{n}\right)^{2},
$$

where $\mu(D, n)$ is the reduced mass of the $B_{0}^{0}$ and nucleon for scattering off a nucleus with atomic number $Z$ and nucleon number $A$. The nucleon parameters can be written as

$$
f_{\text {nucleon }}=\frac{m_{\text {nucleon }}}{v_{246}}\left[\sum_{q=u, d, s} f_{T q}^{\text {(nucleon) }}+\frac{6}{27} f_{T g}^{\text {(nucleon })}\right] .
$$

We have factored out the Higgs coupling to $B_{0}^{0}$, so that only nuclear physics-dependent coefficients are present. Numerically [41],

$$
\begin{array}{ll}
f_{T u}^{(p)}=0.020 & f_{T d}^{(p)}=0.026 \\
f_{T u}^{(n)}=0.014 & f_{T d}^{(n)}=0.036
\end{array}
$$

\footnotetext{
${ }^{4}$ Note that their $h S^{2}$ has a coefficient of $\lambda v_{246}$ which translates into a Feynman rule with coefficient $2 \lambda v_{246}$ to account for identical particles. For a complex scalar, the equivalent Feynman rule constructed from Eq. (4.1) has no factor of 2, and so our $\sigma$ (nucleon) is smaller by a factor of $1 / 4$.
} 
and [42]

$$
f_{T g}^{(p, n)}=1-\sum_{q=u, d, s} f_{T q}^{(p, n)} .
$$

The strange quark contribution to the nucleon is much more uncertain. A recent lattice calculation suggests it is much smaller than has been previously estimated [43] (see also [44]), from which we extract $f_{T s}^{(p, n)} \simeq 0.02$.

Interestingly, since $\mu(D, n) \simeq m_{\text {nucleon }}$, there is essentially no dependence of the nucleon cross section on the mass of the dark matter. This occurs because the (mass) ${ }^{2}$ cancels out between the numerator (its coupling to the Higgs squared) and denominator (from the nonrelativistic expansion of the cross section). Putting all of this together, we obtain

$$
\sigma(\text { nucleon }) \simeq 1.8 \times 10^{-43}\left(\frac{114 \mathrm{GeV}}{m_{h}}\right)^{4} \mathrm{~cm}^{2} .
$$

The current best bounds come from CDMS [19], $\sigma$ (nucleon) $<0.8-3.5 \times 10^{-43} \mathrm{~cm}^{2}$, for dark matter mass between about $200-100 \mathrm{GeV}$. This means that, if the Higgs is very near the LEP bound, quirky dark matter should be seen in direct detection experiments in the very near future. On the other hand, the absence of a direct detection signal would put a lower bound on the Higgs mass that is consistent with quirky dark matter.

\section{Higher dimensional operators}

The interaction of quirky dark matter with nuclei in direct detection experiments can also proceed through higher dimensional operators involving the photon. Since $B_{0}^{0}$ is an electrically neutral scalar composite, all its moments vanish. The leading interactions are then the charge radius and the polarizability operators [45],

$$
L_{\text {elastic }}^{\mathrm{EM}}=\frac{1}{6} e r_{D}^{2} \frac{\partial}{\partial x_{i}} E_{i}+\frac{1}{2} \alpha_{\mathrm{pol}} E^{2},
$$

where $r_{D}$ is the charge radius, and $\alpha_{\text {pol }}$ is the electromagnetic polarizability of $B_{0}^{0}$. These interactions do not scale with the mass ( $A$ number) of the nucleus, and so we cannot use the usual $\sigma$ (nucleon) cross section to compare with experimental results. Instead, we derive the nuclear cross sections that result from the charge radius and polarizability. These nuclear cross sections are in principle easy to compare with experiments, except that experiments often quote bounds only on the average nucleon cross section. Assuming the mass of a detector is dominated by one (heavy) isotope of a nucleus with atomic number $A$, the relationship between the nucleon and the nucleus elastic scattering cross sections are related by

$$
\sigma(\text { nucleus })=\frac{\mu(D, N)^{2}}{\mu(D, n)^{2}} A^{2} \sigma(\text { nucleon })
$$

where $\mu(D, n)$ and $\mu(D, N)$ are the reduced mass of the dark matter with the nucleon and the nucleus, respectively.

\section{Charge radius}

The leading order interaction of a photon to a neutral scalar bound state of charged constituents is given by the charge radius. The scattering cross section off a nucleus due to its charge radius is given by [45]

$$
\sigma(\text { nucleus })_{\text {charge radius }}=\frac{16 \pi}{9} \mu(D, N)^{2} \alpha_{e m}^{2} Z^{2} r_{D}^{4} .
$$

To gain a feeling for the size of the existing constraint, for example, from CDMS, we can compute the bound on $r_{D}^{2}$ for a few specific choices of dark matter mass:

$$
r_{D}^{2} \lesssim \begin{cases}(510 \mathrm{GeV})^{-2} & M=200 \mathrm{GeV} \\ (440 \mathrm{GeV})^{-2} & M=400 \mathrm{GeV} \\ (370 \mathrm{GeV})^{-2} & M=800 \mathrm{GeV} .\end{cases}
$$

We now compute the charge radius of $B_{0}^{0}$ in terms of the mass difference $\delta m_{q}=\left(m_{U}-m_{D}\right)$ and average mass $m_{q}=\left(m_{U}+m_{D}\right) / 2$ of the quirks. The magnitude of the charge radius is estimated by assuming the charge distribution inside the bound state takes the form

$$
\begin{aligned}
\rho(r) & =q e\left(\rho_{U}(r)-\rho_{D}(r)\right) \\
& \approx q e\left[\sum_{i}\left|\sqrt{2}\left(m_{i} \bar{\alpha}\right)^{3 / 2} \exp \left(-m_{i} \bar{\alpha} r\right)\right|^{2}\right],
\end{aligned}
$$

where $\rho_{i}=\left|\left\langle B_{0}^{0} \mid \psi_{i}\right\rangle\right|^{2}$ is the probability density of finding the $i$ th quirk in the bound state $B_{0}^{0}$. We approximated individual quirk wave functions by assuming the other quirk to be fixed in space and normalized it such a way that the total probability of finding the quirk is $1 / 2$. The charge radius, interpreted as a measure of the size of the bound state when probed electromagnetically, is defined classically as the second moment of the spatial charge distribution,

$$
r_{D}^{2}=\frac{1}{e} \int d^{3} r r^{2} \rho(r)=\frac{3 q}{m_{q}^{2} \bar{\alpha}^{2}} \frac{\delta m_{q}}{m_{q}}+\mathcal{O}\left(\frac{\delta m_{q}}{m_{q}}\right)^{2},
$$

where we have assumed $\delta m_{q} \ll m_{q}$. Using the constraint from Eq. (4.11), we find

$$
\frac{\delta m_{q}}{m_{q}} \leq\left(\frac{r_{B}^{-1}}{25 \mathrm{GeV}}\right)^{2} \times \begin{cases}6.4 \times 10^{-3} & M=200 \mathrm{GeV} \\ 8.6 \times 10^{-3} & M=400 \mathrm{GeV} \\ 1.2 \times 10^{-2} & M=800 \mathrm{GeV}\end{cases}
$$

Hence, the quirk masses must be very nearly degenerate to avoid generating an electromagnetic charge radius that exceeds the direct detection bounds. Interestingly, we already required $\delta m_{q}<E_{h f}$, to ensure the lightest quirky baryon is electrically neutral. We see that a self-consistent picture has emerged in which nearly or exactly degenerate quirks ensure both that the lightest quirky baryon is electri- 
cally neutral as well as a negligible electromagnetic charge radius. In Appendix B, we demonstrate that the vanishing of the charge radius can result from imposing an exact discrete symmetry, "UD-parity," which enforces $m_{U}=$ $m_{D}$.

\section{E. Polarizability}

Having discussed and discarded the charge radius operator, we now move on to discuss the electromagnetic polarizability. The scattering cross section due to the polarizability operator is given by [45]

$$
\sigma \text { (nucleus })_{\mathrm{pol}} \approx \frac{144}{25} \mu(D, N)^{2} Z^{4} \alpha_{e m}^{2} \frac{\alpha_{\mathrm{pol}}^{2}}{r_{0}^{2}},
$$

where the nucleus is assumed to be a sphere of homogeneous charge with radius $r_{0}=\sqrt[3]{A} \times 1.2 \mathrm{fm}$. To again gain a feeling for the constraint, for example, from CDMS, we compute the bound on $\alpha_{\text {pol }}$ for a few specific choices of dark matter mass,

$$
\alpha_{\text {pol }} \lesssim \begin{cases}(106 \mathrm{GeV})^{-3} & M=200 \mathrm{GeV} \\ (95 \mathrm{GeV})^{-3} & M=400 \mathrm{GeV} \\ (85 \mathrm{GeV})^{-3} & M=800 \mathrm{GeV}\end{cases}
$$

We now calculate the polarizability of $B_{0}^{0}$. To proceed, we first quickly review the standard polarizability calculation. If an electric field $\mathcal{E}$ is applied to the bound state in the $z$ direction, it causes a perturbation to the Hamiltonian

$$
H_{\text {pert }}=q e \mathcal{E} z
$$

where $q e$ is the constituent quirk charge. The Schrödinger equation in the presence of this perturbation,

$$
\left(H_{0}+H_{\text {pert }}\right)|\psi\rangle=E|\psi\rangle,
$$

can be approximately solved by standard perturbation theory methods,

$$
|\psi\rangle=|0\rangle+q e \mathcal{E} \sum_{k>0} \frac{\langle k|z| 0\rangle}{E_{0}-E_{k}}|k\rangle,
$$

where $H_{0}|k\rangle=E_{k}|k\rangle$, and the ground state is $|0\rangle$. In a moment we will identify the states $\{|k\rangle\}$ with the relevant states of $B_{0}^{0}$. At leading order, the dipole moment of this state in the $z$ direction is composed of two terms:

$$
\begin{aligned}
p_{z}= & -q e\langle\psi|z| \psi\rangle \\
= & -q e\left\{\langle 0|z| 0\rangle+\sum_{k>0}\left[\frac{\langle k|q e \mathcal{E} z| 0\rangle}{E_{0}-E_{k}}\langle 0|z| k\rangle\right.\right. \\
& \left.\left.+\frac{\langle 0|q e \mathcal{E} z| k\rangle}{E_{0}-E_{k}}\langle k|z| 0\rangle\right]\right\} .
\end{aligned}
$$

The first term, $\langle 0|z| 0\rangle$, is the moment of the unperturbed state (if any). The second set of terms represents the moment induced by the electric field, $p_{\text {ind }}=\alpha_{\text {pol }} \mathcal{E}$, where $\alpha_{\text {pol }}$ is defined as the polarizability. We thus find

$$
\alpha_{\text {pol }}=2 q^{2} e^{2} \sum_{k>0} \frac{|\langle k|z| 0\rangle|^{2}}{E_{0}-E_{k}} .
$$

In order to extract the proper dependence of the matrix element in Eq. (4.21) on $\alpha_{e m}$ and $\bar{\alpha}$, we must also include the ordinary electromagnetic Coulomb potential. The unperturbed potential is then simply $V(r)=-(\bar{\alpha}(r)+$ $\left.q^{2} \alpha_{e m}\right) / r$, the effective Bohr radius then is given by $r_{B}=$ $\left[\left(\bar{\alpha}\left(r_{B}\right)+q^{2} \alpha_{e m}\right) \mu\right]^{-1}$ and the energy eigenvalues are given as $E_{k}=-\left(\bar{\alpha}\left(r_{B}\right)+q^{2} \alpha_{e m}\right)^{2} \mu / 2 k^{2}$. Denoting the states by the usual quantum numbers, $\{|k\rangle\}=\{|n, l, m\rangle\}$, with the ground state $|0\rangle=|1,0,0\rangle$, we find

$$
\begin{aligned}
\alpha_{\mathrm{pol}} & =2 q^{2} e^{2} \sum_{n>1} \frac{|\langle n, 1,0|z| 1,0,0\rangle|^{2}}{E_{1}-E_{n}} \\
& =k_{\mathrm{pol}} \frac{9}{2} \frac{q^{2} \alpha_{e m}}{\bar{\alpha}+q^{2} \alpha_{e m}} r_{B}^{3} .
\end{aligned}
$$

The result above shows that there is an additional $q^{2} \alpha_{e m} /\left(\bar{\alpha}+q^{2} \alpha_{e m}\right)$ suppression in the polarizability relative to the hydrogen atom. This agrees with the analogous calculation for the electromagnetic polarizability of heavy quarkonia, substituting the quirkcolor coupling with the QCD coupling [46]. This factor arises since the binding potential is proportional to $\bar{\alpha}+q^{2} \alpha_{e m}$. The additional non-Abelian correction resulting from the $\log r$ term in the potential is encoded in the coefficient $k_{\mathrm{pol}}$. Numerically solving the Schrödinger equation, we calculated the corrections to the first few terms, finding the largest correction to the $\langle 2,1,0|z| 1,0,0\rangle$ term and a smaller correction to the $\langle 3,1,0|z| 1,0,0\rangle$ term. Extrapolating from these results, our numerical estimate is $k_{\mathrm{pol}}=$ $(1.3,1.4,1.5)$ for $\bar{\alpha}\left(r_{B}\right)=(0.2,0.3,0.4)$ and $N_{f}=2$.

We can now easily determine the bounds and prospects for detection of quirky dark matter as a function of $\bar{\alpha}$ using our expression for $\alpha_{\text {pol }}$ in Eq. (4.22), In Fig. 3 we show theory predictions and experimental bounds on an effective cross section. To combine results from several experiments with theory predictions, the effective nuclear cross section applicable to the polarizability operator is

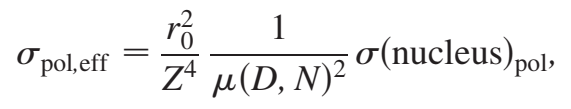

where the individual experiments' target size $\left(r_{0}\right)$ and charge ( $Z$ value) have been factored out, along with two powers of the reduced mass, analogous to Eq. (4.9).

To gain an appreciation of the constraint on $r_{B}^{-1}$, we can compute the lower bounds on the inverse Bohr radius derived from the polarizability constraint directly from Eqs. (4.22) and (4.16):

$$
r_{B}^{-1} \gtrsim \begin{cases}40-32 \mathrm{GeV} & M=200 \mathrm{GeV} \\ 33-27 \mathrm{GeV} & M=400 \mathrm{GeV} \\ 30-24 \mathrm{GeV} & M=800 \mathrm{GeV}\end{cases}
$$




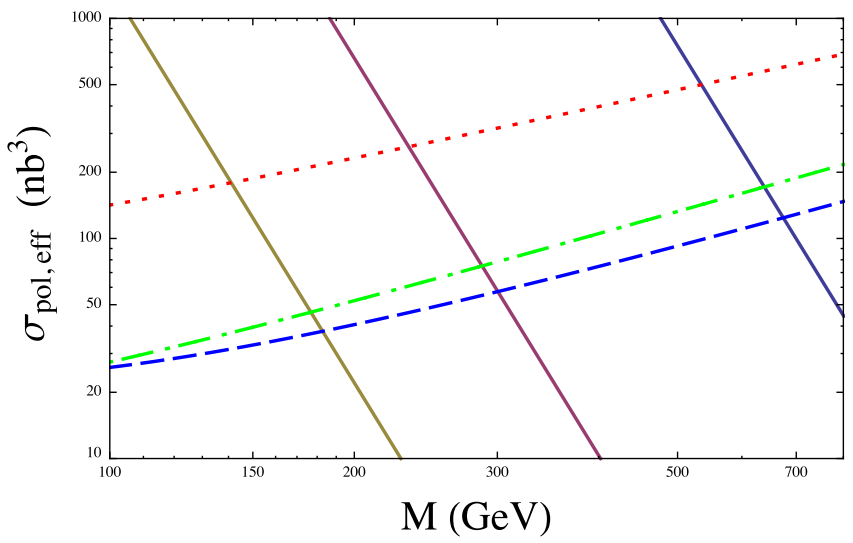

FIG. 3 (color online). Bounds of nuclear cross section. We have plotted $\sigma_{\text {pol,eff }}$ from Eq. (4.23) using data from CDMS (blue dashed line), xenon (green dot-dashed line) and tungsten (red dotted line). The solid lines are theory predictions due to the polarizability operator for two flavors as a function of dark matter mass $M$. From right to left we have plotted $\sigma_{\text {eff }}$ for $\bar{\alpha}\left(r_{B}\right)=\{0.2$ (blue), 0.4(purple), 0.6(yellow) $\}$, respectively.

where the range in value corresponds to $0.2<\bar{\alpha}<0.6$. Since the cross section induced by the electromagnetic polarizability is proportional to $r_{B}^{6}$, there is a relatively minor dependence on the mass of dark matter and the strength of the strong force. The large mass point $M=$ 800 (as well as the larger masses shown in Fig. 3) implies large quirk masses $m_{q} \simeq 400 \mathrm{GeV}$, with correspondingly large Yukawa couplings, $\lambda_{q} \simeq 2.2$. This is shown for comparison with the lighter masses. We do not address the semiperturbative nature of these Yukawa couplings.

\section{INDIRECT DETECTION THROUGH ABSORPTION LINES}

An interesting feature of quirky dark matter is that it has a spectrum of excited bound states that can be excited by absorption of SM particles such as the photon and $Z$. The photon interaction, in particular, provides a fascinating possibility to probe dark matter directly through photon absorption lines, entirely analogous to how matter itself is probed through its own photon absorption lines. The typical energies for quirky excitations, as we see below, are in the gamma-ray region. The possible existence of dark lines due to inelastic transitions of dark matter was considered before in a somewhat different setup in Ref. [47]. We will apply their results, suitably modified for our case (with one correction to their formulae given in Appendix C), to the two transitions of greatest interest: the quirky Lyman-alpha transition, and the quirky hyperfine transition, illustrated in Fig. 1.

\section{A. Quirky Lyman-alpha}

The photon energy corresponding to quirky Lymanalpha transition, between the ground state and the first excited state, can be read off from Eq. (2.5),

$$
E_{L \alpha}=E_{2}-E_{1}=\frac{4 k_{1}-k_{2}}{3} \frac{8}{3} \bar{\alpha}^{2} \mu .
$$

The deviation from purely Coulombic is characterized by the constant $\left(4 k_{1}-k_{2}\right) / 3 \simeq(1.0,1.0,0.9)$ for $\bar{\alpha}\left(r_{B}\right)=$ $(0.2,0.3,0.4)$, which is negligible for the quirky Lymanalpha transition.

The width of this absorption feature can be computed in the Coulombic approximation by analogy with hydrogen Lyman-alpha. We obtain

$$
\Gamma_{L \alpha}=\frac{4}{9} q^{2} \alpha_{e m} E_{L \alpha}^{3}|\langle 1|\bar{r}| 0\rangle|^{2}=\frac{64}{6561} \alpha_{e m} \bar{\alpha}^{4} \mu .
$$

This width, taking $\bar{\alpha} \rightarrow \alpha_{e m}$ and $q \rightarrow 1$ reproduces the well-known value for hydrogen Lyman-alpha. Plugging in representative values, the width is

$$
\Gamma_{L \alpha}=5 \times 10^{-4} \mathrm{GeV}\left(\frac{\bar{\alpha}}{0.4}\right)^{3}\left(\frac{r_{B}^{-1}}{25 \mathrm{GeV}}\right) .
$$

It will also prove convenient to express the width as

$$
\frac{\Gamma_{L \alpha}}{E_{L \alpha}}=\frac{512}{19683} \alpha_{e m} \bar{\alpha}^{2} \simeq 1.9 \times 10^{-4} \bar{\alpha}^{2} .
$$

\section{B. Quirky hyperfine}

The next transition of interest arises from the quirky hyperfine structure. The energy splitting between the $B_{1}^{0}$ and $B_{0}^{0}$ states is given by

$$
E_{h f}=c_{1,0} \frac{2}{3} \bar{\alpha}^{4} \mu,
$$

where $c_{1,0}$ is an order one constant that characterizes the deviation from purely Coulombic. A few specific values were computed in Sec. II B.

The decay rate of the quirky hyperfine transition can also be computed. The result in the purely Coulombic approximation is the same as for quarkonia [48]:

$$
\Gamma_{h f}=\frac{4}{3} \alpha_{e m} q^{2} \frac{E_{h f}^{3}}{\mu^{2}}=\frac{8}{81} \alpha_{e m} \bar{\alpha}^{12} \mu .
$$

Plugging in representative values, we find the width to be

$$
\Gamma_{h f}=8 \times 10^{-7} \mathrm{GeV}\left(\frac{\bar{\alpha}}{0.4}\right)^{11}\left(\frac{r_{B}^{-1}}{25 \mathrm{GeV}}\right) .
$$

It will again prove convenient to express the width as

$$
\frac{\Gamma_{h f}}{E_{h f}}=\frac{4}{27} \alpha_{e m} \bar{\alpha}^{8} \simeq 1.1 \times 10^{-3} \bar{\alpha}^{8} .
$$

\section{Absorption and broadening}

For these transitions to be visible to gamma-ray observatories, three things are required: (1) the cross section on resonance should be substantial, (2) the width of the dark 
matter Doppler-broadened resonance should be resolvable, and (3) the energy of the transition should be within the range that is observable. Several observatories view the universe in the relevant energy range, satisfying (3). In this section we compute the cross section and Dopplerbroadened width.

The absorption cross section near a resonance takes the usual Breit-Wigner form [49]

$$
\sigma_{\mathrm{abs}}=\frac{6 \pi}{p_{\mathrm{c} . \mathrm{m} .}^{2}} \frac{M_{*}^{2} \Gamma_{*}^{2}}{\left(s-M_{*}^{2}\right)^{2}+M_{*}^{2} \Gamma_{*}^{2}},
$$

where $M$ is the mass of the ground state (the mass of quirky dark matter), and $M_{*}=M+E_{\gamma}$ is the mass of the excited state. The dominant decay of the resonance is into photons, $\Gamma_{*} \simeq \Gamma_{M_{*} \rightarrow M \gamma}$, since decay to an on-shell $Z$ is kinematically forbidden (throughout our parameter space), while decay through a virtual photon to a fermion pair is suppressed by $\alpha_{e m}$. The factor of $6 \pi$ comes from $4 \pi\left(2 j_{R}+\right.$ $1) /\left(\left(2 s_{1}+1\right)\left(2 s_{2}+1\right)\right.$ where $j_{R}=1$ is the massive spin- 1 resonance, $\left(2 s_{1}+1\right)=2$ for the massless photon, and $s_{2}=0$ for the massive scalar quirky dark matter particle.

There are two potential methods to exploit this absorption cross section. The first, and most promising, is to look towards massive galaxy clusters that have the largest column density of dark matter and a large dark matter velocity dispersion. We then compare this to the seemingly less promising method of looking for absorption over cosmological distances.

We follow the formalism of [47] to determine the effect of the broadening and the size of the absorption cross section. This formalism applies to any of the photoninduced baryonic excitations, including quirky Lymanalpha and quirky hyperfine. The resonance energy is

$$
E_{\gamma}^{\mathrm{res}}=\frac{M_{*}^{2}-M^{2}}{2 M}
$$

which is roughly equal to the mass difference, $M_{*}-M$ for $\bar{\alpha} \lesssim 1$.

The dark matter velocity distribution within a galaxy cluster is assumed to follow a Maxwell-Boltzmann velocity distribution, denoted $f_{\mathrm{MB}}(p)$. The effective absorption cross section for a photon of energy $E_{\gamma}$ is

$$
\sigma\left(E_{\gamma}\right)=\int_{0}^{\infty} d p f_{\mathrm{MB}}(p)\langle\sigma\rangle,
$$

where

$$
\langle\sigma\rangle=\int_{-1}^{1} \frac{d \cos \theta}{2} \frac{6 \pi}{p_{\mathrm{c} . \mathrm{m} .}^{2}} \frac{M_{*}^{2} \Gamma_{*}^{2}}{\left(s-M_{*}^{2}\right)^{2}+M_{*}^{2} \Gamma_{*}^{2}},
$$

is the total cross section after integrating over the incident angle. The center-of-mass energy is $s=M^{2}+$ $2 E_{\gamma}\left(\sqrt{p^{2}+M^{2}}-p \cos \theta\right)$ while the momentum in the center-of-mass frame is given by

$$
p_{\mathrm{c} . \mathrm{m} .}^{2}=\frac{\left(M^{2}-s\right)^{2}}{4 s} .
$$

The integral in Eq. (5.12) was solved analytically in the Appendix of Ref. [47], which we checked and agree with except for a correction to one expression given in our Appendix C.

For our case, with $M_{*} \simeq M \gg E_{\gamma}$, the analytic formula can be drastically simplified in the following limits. First, observe that $M_{*} \Gamma_{*} \ll \Delta M^{2}$ which is equivalent to $2 \Gamma_{*} \ll$ $E_{\gamma}^{\text {res }}$, is automatic for our perturbative calculation of the width of the resonance to be valid. Next, consider the limit

$$
\Gamma_{*} M_{*} \ll 2 E_{\gamma}^{\mathrm{res}} p
$$

which corresponds to roughly

$$
\Gamma_{*} \ll 2 E_{\gamma}^{\mathrm{res}} \sigma_{v}
$$

given the average momentum of dark matter with a Maxwell-Boltzmann distribution is roughly $p \simeq M \sigma_{v}$. Now compare this expression with Eqs. (5.4) and (5.8). For $\bar{\alpha} \lesssim 1$, Eq. (5.15) is satisfied for $\sigma_{v} \gtrsim 10^{-4}$, which is itself satisfied by large galaxy clusters.

Putting all this together, we obtain the following simple formula for the cross section on resonance,

$$
\left.\langle\sigma\rangle\right|_{\mathrm{res}}=\frac{3}{2} \pi^{2} \frac{\Gamma_{*} M}{E_{\gamma}^{\mathrm{res}^{3}} p},
$$

which can be integrated over a Maxwell-Boltzmann distribution to become

$$
\sigma\left(E_{\gamma}=E_{\gamma}^{\mathrm{res}}\right)=\frac{3}{\sqrt{2}} \pi^{3 / 2} \frac{\Gamma_{*}}{E_{\gamma}^{\mathrm{res}^{3}} \sigma_{v}} .
$$

Interestingly, this form of the on-resonance cross section has several important features: First, there is no explicit dependence on the mass of the particle. Second, the resonance photon energy dependence in $\Gamma_{*}$ (from Lyman-alpha or hyperfine) exactly cancels the dependence in the denominator. This leads to very simple expressions for the on-resonance absorption cross section

$$
\begin{gathered}
\sigma\left(E_{\gamma}=E_{\gamma}^{\mathrm{res}}\right)=\frac{16384 \sqrt{2} \pi^{3 / 2}}{59049} \alpha_{e m} \frac{r_{B}^{2}}{\sigma_{v}} \quad(L \alpha \\
\sigma\left(E_{\gamma}=E_{\gamma}^{\mathrm{res}}\right)=\frac{\pi^{3 / 2}}{\sqrt{2}} \alpha_{e m} \bar{\alpha}^{2} \frac{r_{B}^{2}}{\sigma_{v}} \quad(h f) .
\end{gathered}
$$

The opacity to $\gamma$ rays due to these dark lines on resonance can be estimated using the optical depth $\tau=\sigma \Sigma_{\mathrm{DM}} / M$, where $\Sigma_{\mathrm{DM}}$ is the dark matter surface density associated with the integral along the line of sight of the dark matter density. As an example, consider the Coma cluster, which has a surface density that was estimated by Ref. [47] to be $\Sigma_{\mathrm{DM}} \simeq 5 \times 10^{26} \mathrm{GeV} / \mathrm{cm}^{2}$ with velocity dispersion $\sigma_{v}=$ $820 \mathrm{~km} / \mathrm{s}$. Plugging in these characteristic values, 


$$
\begin{aligned}
\left.\tau\right|_{\mathrm{res}}= & 1 \times 10^{-5} \frac{820 \mathrm{~km} / \mathrm{s}}{\sigma_{v}}\left(\frac{25 \mathrm{GeV}}{r_{B}^{-1}}\right)^{2} \frac{200 \mathrm{GeV}}{M} \\
& \times \frac{\Sigma_{\mathrm{DM}}}{5 \times 10^{26} \mathrm{GeV} / \mathrm{cm}^{2}} \quad(L \alpha) \\
\left.\tau\right|_{\mathrm{res}}= & 1.5 \times 10^{-5} \bar{\alpha}^{2} \frac{820 \mathrm{~km} / \mathrm{s}}{\sigma_{v}}\left(\frac{25 \mathrm{GeV}}{r_{B}^{-1}}\right)^{2} \frac{200 \mathrm{GeV}}{M} \\
& \times \frac{\Sigma_{\mathrm{DM}}}{5 \times 10^{26} \mathrm{GeV} / \mathrm{cm}^{2}}(\text { hf }),
\end{aligned}
$$

we see that $\tau$ is much smaller than 1 . For the Coma cluster, only a small fraction of photons are expected to be absorbed in either the Lyman-alpha or hyperfine transitions.

There are three potential ways to improve on this result. The first is to search for systems with larger surface mass densities. This is most likely to arise in the largest clusters that are also the most compact, and thus have a very concentrated mass function. The second is to perform more precise measurements of the photon flux of particular clusters, which would allow probing optical depths considerably smaller than 1 . Third, combining $\gamma$-ray spectra from many different clusters of different mass, velocity dispersion, and redshift, and suitably processing them into a normalizable spectrum, one could significantly improve the search for dark lines through large scale galaxy cluster sampling. Given that the cluster number density is rising rapidly as the cluster mass is decreased (see, e.g., [50]), and with improved photon flux and energy resolution, this is probably the best approach for the future.

Assuming methods are developed to effectively probe these small optical depths, it is also important to know the Doppler-broadened resonance width. In our case, the Doppler-broadened width arises from the dark matter velocity distribution in the observed systems. For a MaxwellBoltzmann velocity distribution, we find the observed width of the resonance is well fit to a Gaussian

$$
\sigma\left(E_{\gamma}\right)=\sigma\left(E_{\gamma}^{\mathrm{res}}\right) \exp \left[-\frac{\left(E_{\gamma}-E_{\gamma}^{\mathrm{res}}\right)^{2}}{2\left(\sigma_{v} E_{\gamma}^{\text {res }}\right)^{2}}\right]
$$

with a fractional width $\Delta E / E \simeq \sigma_{v}$.

The velocity dispersion of dark matter in galaxy clusters has been found to scale as [51]

$$
\sigma_{v} \simeq(1080 \mathrm{~km} / \mathrm{s})\left(\frac{h(z) M_{\text {clus }}}{10^{15} M_{\odot}}\right)^{0.336},
$$

where $h(z)=H(z) / 100 \mathrm{~km} \mathrm{~s}^{-1} \mathrm{Mpc}^{-1}$ is the normalized Hubble parameter at redshift $z$ and $M_{\text {clus }}$ is the cluster mass (defined as the mass within a sphere encompassing a mean mass density of 200 times the background matter density at that redshift). Searching for dark lines of dark matter in clusters thus requires the gamma-ray energy resolution comparable to the velocity dispersion. The full width at half maximum (FWHM) is approximately $2.35 \sigma_{v} \simeq$ $0.003-0.01$ for the largest galaxy clusters with mass between about $10^{14-15} M_{\odot}$. Interestingly, for soft $\gamma$ rays up to $8 \mathrm{MeV}$, this resolution was achieved with the INTEGRAL spectrometer [52]. The EGRET and Fermi/GLAST observatories extend up to much higher gamma-ray energies, $30 \mathrm{GeV}$ and about $1 \mathrm{TeV}$, respectively. Unfortunately, the FWHM energy resolution of these instruments is roughly 0.2 for EGRET and between $0.05-0.1$ for Fermi/GLAST, which is a just bit too course to likely resolve the $\gamma$-ray dark line feature that we predict in our model.

Finally, we consider the effect of scattering over cosmological distances. At a redshift of $z \sim 1$ or larger, a photon with a given initial energy $E_{\gamma}$ will sweep out a resonance of width $\Gamma \sim z_{\gamma}$ (e.g. $[53,54]$ ). In order for this to give an observable effect, however, the mean-free path $\ell$ for photon absorption must be shorter than the cosmological distance that the photon spends on resonance:

$$
\ell=\frac{M}{\left.\rho_{D}\langle\sigma\rangle\right|_{\mathrm{res}}} \lesssim H^{-1} \frac{\Gamma}{E_{\gamma}} .
$$

Dark matter in the cosmos has negligible momentum, and so the resonance cross section can be obtained from Eq. (5.9) in the limit $p \ll M,\left.\langle\sigma\rangle\right|_{\text {res }}=6 \pi / p_{\text {c.m. }}^{2}=$ $6 \pi / E_{\gamma}^{2}$. Substituting, we obtain

$$
\frac{\ell}{H^{-1}} \simeq 2 \times 10^{4} \frac{M}{200 \mathrm{GeV}}\left(\frac{E_{\gamma}}{100 \mathrm{MeV}}\right)^{2},
$$

which shows that, even if $\Gamma \sim E_{\gamma}$, the photon does not travel nearly far enough to be absorbed over a cosmological distance.

\section{QUIRKCOLOR GLUEBALL DECAY}

With exactly two flavors, our quirkcolor theory confines. It is straightforward to estimate the confinement scale $\Lambda_{Q}$. Quirky theories suffer from a potential cosmological problem, namely, photon injection during nucleosynthesis, if glueballs decay into photons with a lifetime that is of order $1 \mathrm{~s}$.

Confinement gives mass to the quirkcolor "glueballs" that we crudely approximate to have mass $\Lambda_{Q}$. For theories with vectorlike quirks, Refs. [22,28] showed that the glueballs decay slowly, since the leading operators are suppressed by many powers of the quirk mass. One such operator is

$$
\frac{q^{2} \alpha_{e m} \bar{\alpha}\left(m_{q}\right)}{m_{q}^{4}} F_{Q}^{\mu \nu} F_{Q \mu \nu} F^{\rho \sigma} F_{\rho \sigma}
$$

where $F_{Q}$ and $F$ are the field strengths of the quirkcolor group and electromagnetism, respectively. This results in a decay rate 


$$
\begin{aligned}
\Gamma & \sim \sum_{\text {quirks }} \frac{q^{4} \alpha_{\mathrm{em}}^{2} \bar{\alpha}\left(m_{q}\right)^{2}}{8 \pi} \frac{\Lambda_{Q}^{9}}{m_{q}^{8}} \\
& \simeq\left(\frac{\Lambda_{Q}}{1 \mathrm{GeV}}\right)^{9}\left(\frac{100 \mathrm{GeV}}{m_{q}}\right)^{8}\left(\frac{\bar{\alpha}\left(m_{q}\right)}{0.1}\right)^{2} \mathrm{~s}^{-1},
\end{aligned}
$$

where the sum is over all of the quirks given in Table I.

A different operator exists in our model due to the Higgs coupling to our chiral quirks. Integrating out quirks and the scalar Higgs boson simultaneously results in a dimension-7 operator,

$$
\frac{\bar{\alpha}\left(m_{q}\right) m_{f}}{4 \pi v^{2} m_{h}^{2}} F_{Q}^{\mu \nu} F_{Q \mu \nu} \bar{f} f
$$

where $v=174 \mathrm{GeV}$. This leads to glueball decay into a pair of light SM fermions that satisfies $2 m_{f} \lesssim \Lambda_{Q}$. Despite the lower dimensionality, this operator is not obviously less suppressed than Eq. (6.1), due to the Yukawa suppression $m_{f} / v$. The decay rate is

$$
\begin{aligned}
\Gamma & \sim \sum_{\text {quirks }} \frac{\bar{\alpha}\left(m_{q}\right)^{2} m_{f}^{2}}{8 \pi} \frac{\Lambda_{Q}^{7}}{v^{4} m_{h}^{4}} \\
& \simeq\left(\frac{\Lambda_{Q}}{1 \mathrm{GeV}}\right)^{7}\left(\frac{115 \mathrm{GeV}}{m_{h}}\right)^{4}\left(\frac{\bar{\alpha}\left(m_{q}\right)}{0.1}\right)^{2}\left(\frac{m_{f}}{0.1 \mathrm{GeV}}\right)^{2} \mathrm{~s}^{-1} .
\end{aligned}
$$

We see this decay rate is comparable to the rate into photons for the example parameters. The main distinction we draw is that the Higgs-mediated decay does not depend on the quirk mass. Hence, we can contemplate quirk masses that significantly exceed $100 \mathrm{GeV}$ without necessarily leading to cosmological difficulties of late decaying glueballs, so long as the Higgs is relatively light. We emphasize that, while our estimates are parametrically correct, they nevertheless have significant uncertainties, particularly with regard to the identification of the glueball mass with $\Lambda_{Q}$.

\section{DISCUSSION}

We have presented a new theory of dark matter that is made up of a baryonic composite of a quirks that transform under a new strongly coupled sector, $S U(2)_{Q}$ quirkcolor. The abundance of quirky dark matter is linked to the baryonic abundance through electroweak sphalerons.

The baryonic excitation spectrum was computed, including the fine and hyperfine structure. The lightest baryonic state can be made automatically charged neutral when the quirks have (nearly) degenerate masses, which can be ensured through a discrete symmetry (UD parity). Degenerate quirk masses also eliminate the dimension-6 electromagnetic charge radius operator, allowing a much larger range of scales to be considered.
Quirky dark matter is at the threshold of direct direction through elastic nuclear recoil. Two processes lead to nuclear recoil cross sections through (i) Higgs exchange, which couples proportional to the (mass) ${ }^{2}$ of the nuclei (as usual), and (ii) electromagnetic polarizability, which couples to the electric charge $Z^{4}$.

Indirect detection may be possible by searching for a gamma-ray "dark line" spectroscopic feature in galaxy clusters that results from the quirky Lyman-alpha or quirky hyperfine transitions. This is a difficult measurement that might be possible in the future. It requires sensitivity to optical depths much smaller than 1 . We envision this could be accomplished with excellent gamma-ray spectral sensitivity applied to a large number of galaxy clusters, suitably combining the results together. The feature itself has a Doppler-broadened FWHM roughly of order $2.35 \Delta E_{\gamma} / E_{\gamma} \simeq 2.35 \sigma_{v} \simeq 0.003-0.01$. This is close to but somewhat smaller than the FWHM energy resolution of EGRET and Fermi. Indirect detection through other means, such as annihilation in the Sun, galaxy, or beyond, does not occur so long as the full global $U(1)_{\mathrm{QB}}$ quirky baryon number is exact. Annihilation signals would reappear if quirky baryon number were broken to a $Z_{2}$, and signals of dark matter decay would result if $U(1)_{\mathrm{QB}}$ were completely broken (but only very, very slightly).

The collider signals of quirky dark matter represent a plethora of possibilities [22] (see also [55]). Quirks can be pair produced, which form bound states with quirkcolor strings attached. They will stretch and flop back and forth shedding angular momentum in some combination of quirky glueballs (from the quirks and the quirkcolor string) as well as photon emission from the quirks which may result in interesting underlying event signals [56]. Eventually the quirks bound in a mesonic state recombine and annihilate, somewhat analogous to heavy quarkonia annihilation. Quirky baryon production is kinematically suppressed due to the need to pair produce a baryon and antibaryon (four quirks total), to conserve quirky baryon number. Clearly, the collider physics of quirky dark matter is an area ripe for future exploration.

\section{ACKNOWLEDGMENTS}

We thank R. Essig, R. Harnik, M. Luty, A. Nelson, L. Strigari, and J. Wacker for helpful discussions at various stages of this work. We also thank the Aspen Center for Physics, the Yukawa Institute of Theoretical Physics, the Kavli Institute of Theoretical Physics, and the Galileo Galilei Institute for Theoretical Physics for hospitality where part of this work was completed. This work was supported in part by the U.S. Department of Energy under Contracts No. DE-FG02-96ER40969 (G.D.K. and T. S. R.), No. DE-FG03-91ER40674 and No. DE-FG0295ER40896 (K. M. R.), No. DE-FG02-91ER40674 (J. T.), and by the NSF under Contract No. PHY-0918108 (G. D. K.). 


\section{APPENDIX A: NONRELATIVISTIC DETAILS}

The construction of the nonrelativistic theory starting from the theory described in Sec. II A begins with the chiral two-component spinors in Table I. Below the electroweak scale, the two-component fermions can be written in terms of two four-component Dirac fermions and their charge conjugates for the quirks and the antiquirks, respectively,

$$
\begin{aligned}
f_{U} \equiv\left(\begin{array}{c}
\xi_{U} \\
\xi_{\bar{U}}^{\dagger}
\end{array}\right), \quad f_{D} & \equiv\left(\begin{array}{c}
\xi_{D} \\
\xi_{\bar{D}}^{\dagger}
\end{array}\right), \quad \text { and } f_{a}^{c}=i \gamma_{0} \gamma_{2} f_{a}^{T} \\
\mathcal{L}_{m} & =\sum_{i} m_{i} \bar{f}_{i} f_{i},
\end{aligned}
$$

where $i$ runs over the flavor indices $\{U, D\}$. In this basis, the four-component Dirac spinors constructed are in the Weyl basis, which makes chirality manifest and is the most convenient choice for representing a relativistic chiral theory. In a nonrelativistic theory, however, the Dirac basis is more suitable. The $\gamma$ matrices and all the fourcomponent spinors can be rotated from the Weyl basis to the Dirac basis by the transformation

$$
\begin{gathered}
\gamma_{\mu} \rightarrow U \gamma_{\mu} U^{\dagger} \text { and } f_{i} \rightarrow U f_{i}, \\
\text { where } U=\frac{1}{\sqrt{2}}\left(\begin{array}{cc}
1 & 1 \\
-1 & 1
\end{array}\right) .
\end{gathered}
$$

The advantage of this basis is that $\gamma_{0}=\operatorname{diag}(1,1,1,1)$ is diagonal, and so the dominant component of the Dirac four spinor [namely, $\left(1+\gamma_{0}\right) f_{i}$ ], is a two-component Pauli spinor, which furnishes the minimal representation for the nonrelativistic spinor field.

The next step is to eliminate the quirk mass scales $m_{i}$ while keeping the heavy quirk fields. This is accomplished by a simple time-dependent rescaling of the Dirac fermions in Eq. (A2). There is now a preferred frame, namely, the center-of-mass frame, which is the frame in which we work from now on. Hence, the dominant component of the full Dirac spinors become

$$
\begin{gathered}
\psi_{i}=e^{i m_{i} t \frac{1}{2}\left(1+\gamma_{0}\right) f_{i}} \\
\chi_{i}=e^{-i m_{i} t \frac{1}{2}\left(1+\gamma_{0}\right) f_{i}^{c} .}
\end{gathered}
$$

The field $\psi$ annihilates a heavy quirk field, while $\chi$ creates a heavy antiquirk field. These spinors roughly correspond to particle and antiparticle and are appropriate for a nonrelativistic approximation about the center-of-mass frame of reference. Classically, the entire quirk and the antiquirk Dirac spinors in Eq. (A1) can be written in terms of the spinors in Eqs. (A3) and (A4):

$f_{i}=e^{-i m_{i} t}\left(\begin{array}{c}\psi_{i} \\ \frac{i \vec{\sigma} \cdot \vec{D}}{2 m_{i}+i D_{0}} \psi_{i}\end{array}\right) \quad$ and $f_{i}^{c}=e^{i m_{i} t}\left(\begin{array}{c}\chi_{i} \\ \frac{i \vec{\sigma} \cdot \vec{D}}{2 m_{i}+i D_{0}} \chi_{i}\end{array}\right)$.
The nonrelativistic Lagrangian is written in terms of the spinors $\psi$ and $\chi$ that designate almost on-shell quirks and antiquirks. The Lagrangian can now be computed as an expansion in $1 / m_{i}$ [57]:

$$
\begin{aligned}
\mathcal{L}_{0}^{\mathrm{NR}}= & \sum_{i} \psi_{i}^{\dagger}\left(i D_{0}+\frac{1}{2 m_{i}} \vec{D}^{2}\right) \psi_{i}+\frac{c_{F} g_{q}}{2 m_{i}} \psi_{i}^{\dagger} \vec{\sigma} \cdot \vec{B}_{q} \psi_{i} \\
& +\frac{g_{q} c_{D}}{8 m_{i}^{2}} \psi_{i}^{\dagger}\left(\vec{D} \cdot \vec{E}_{q}-\vec{E}_{q} \cdot \vec{D}\right) \psi_{i} \\
& +\frac{g_{q} c_{s}}{8 m_{i}^{2}} \psi_{i}^{\dagger} \vec{\sigma} \cdot\left(\vec{D} \times \vec{E}_{q}-\vec{E}_{q} \times \vec{D}\right) \psi_{i} \\
& -\left(\psi_{i} \leftrightarrow \chi_{i}\right)+\mathcal{O}\left(\frac{1}{m^{3}}\right),
\end{aligned}
$$

where $i D_{0}=i \partial_{0}-g_{q} A_{q_{0}}, i \vec{D}=i \vec{\nabla}+i g_{q} \vec{A}_{q}$. The electric and magnetic quirky gauge fields are defined as usual $E_{q_{i}}=F_{q_{0 i}}$ and $B_{q_{i}}=\epsilon_{i j k} F_{q}^{j k}$. At tree level, the matching is simply $c_{F}=c_{D}=c_{s}=1$. This is corrected due to quantum effects by $\mathcal{O}\left(g_{q}^{2}\right)$. The terms in this Lagrangian in Eq. (A6) have well-known physical interpretation. The $\vec{\sigma} \cdot \vec{B}_{q}$ term is the quirkcolor-magnetic moment interaction, the $\vec{D} \cdot \vec{E}_{q}$ term is the Darwin term, and the $\vec{D} \times \vec{E}_{q}$ term is the spin-orbit coupling. The $1 / \mathrm{m}^{3}$ term contains the first relativistic correction.

\section{Spectrum}

The ground state spectrum of baryons and mesons can be determined by cataloging the Lorentz invariant bilinears made out of two $\psi$ 's or one $\psi$ and one $\chi$, respectively. The decomposition of the fermion bilinears in (flavor, spin) space can be written as

$$
(\mathbf{2}, \mathbf{2}) \otimes(\mathbf{2}, \mathbf{2})=\left(\mathbf{1}_{a}, \mathbf{1}_{a}\right) \oplus\left(\mathbf{3}_{s}, \mathbf{1}_{a}\right) \oplus\left(\mathbf{1}_{a}, \mathbf{3}_{s}\right) \oplus\left(\mathbf{3}_{s}, \mathbf{3}_{s}\right),
$$

where the subscripts $s$ and $a$ denote the symmetric and antisymmetric representation, respectively. Mesons can be written in all of these representations since they are formed of nonidentical particles. Baryons, however, must satisfy the Pauli exclusion principle. Combining two identical quirks into a Lorentz invariant requires an antisymmetric $S U(2)_{Q}$ contraction, and consequently, only totally symmetric combinations of (flavor, spin) are possible, specifically, $\left(\mathbf{1}_{a}, \mathbf{1}_{a}\right)$ and $\left(\mathbf{3}_{s}, \mathbf{3}_{s}\right)$. The resulting baryonic states can be written as

$$
\begin{aligned}
\left(\mathbf{1}_{a}, \mathbf{1}_{a}\right): B_{0}^{0} & =\tilde{\psi}^{i \alpha} \psi_{i \alpha} \\
\left(\mathbf{3}_{s}, \mathbf{3}_{s}\right): B^{F S} & =\tilde{\psi}^{i \alpha}\left(\sigma^{F}\right)_{i}{ }^{j}\left(\sigma^{S}\right)_{\alpha}{ }^{\beta} \psi_{j \beta},
\end{aligned}
$$

in terms of $\tilde{\psi}^{i \alpha} \equiv \epsilon^{i j} \epsilon^{\alpha \beta} \psi_{j \beta}^{T}$. Here, flavor indices are designated by Latin letters $(i, j$, etc.), spin by Greek indices ( $\alpha, \beta$, etc.), and quirkcolor indices have been suppressed. The resulting states consist of $B_{0}^{0}$, a complex scalar with zero electric charge and +1 baryon charge, and $B^{F S}$, a 
massive spin-1 vector that is triplet under flavor and also carries +1 baryon charge. The antibaryons with opposite baryon charge are similarly constructed. The $B^{F S}$ can be decomposed in terms of spin-1 baryons with electric charge $q$, denoted by $B_{1}^{q}$ (suppressing the spin index $S$ ), as

$$
\begin{gathered}
B_{1}^{0} \equiv B^{3 S} \\
B_{1}^{ \pm} \equiv \frac{1}{\sqrt{2}}\left(B^{1 S} \mp i B^{2 S}\right) .
\end{gathered}
$$

The mesons are made of $\chi$ and $\psi$ in representations given by Eq. (A7). In rough analogy to mesons in QCD with two flavors, we can write the mesons as

$$
\begin{aligned}
& \left(\mathbf{1}_{a}, \mathbf{1}_{a}\right): \eta=\tilde{\psi} \chi \\
& \left(\mathbf{3}_{s}, \mathbf{1}_{a}\right): \pi=\tilde{\psi} \sigma^{F} \chi \\
& \left(\mathbf{1}_{s}, \mathbf{3}_{a}\right): \phi=\tilde{\psi} \sigma^{S} \chi \\
& \left(\mathbf{3}_{s}, \mathbf{3}_{s}\right): \rho=\tilde{\psi} \sigma^{F} \sigma^{S} \chi .
\end{aligned}
$$

We have suppressed all fermion indices. The proper combinations of flavor states that yield definite electric charge states are formed analogously to Eq. (A11).

\section{Nonrelativistic bound states in quantum quirky dynamics}

We assume that the quirkcolor force is weakly coupled and thus described by perturbative physics with the quirks much heavier than the resulting bound state energies. In this limit, the time scale of the relative heavy quirk movement becomes much larger than the time scale of the quirky gluon dynamics. Then, feedback effects of the moving heavy quirks on gluons can be neglected, and so the adiabatic approximation or the leading BornOppenheimer approximation should be applicable. Also, one can use the different energy scales just like in the positronium problem in QED (discarding the annihilation effect). The nonrelativistic bound state is characterized by the scale of the quirk mass $m$ (hard), the scale of the momentum transfer $p \sim m v$ (soft), and the scale of kinetic energy of the quirks in the center-of-mass frame $E \sim$ $p^{2} / m \sim m v^{2}$ (ultrasoft), where $v$ is the heavy quirk velocity in this frame. In our weakly coupled nonrelativistic system, $v \sim \alpha_{q} \ll 1$ and it follows that the three relevant scales are hierarchically ordered (i.e. $m \gg m v \gg m v^{2}$ ).

Interestingly, such a situation also happens in quarkonium physics, where the same hierarchy has been utilized to construct equivalent effective theories to describe the quarkonium spectra and interactions (for some reviews, see e.g. [58-66]). First, the transition is from the EFT with relativistic quarks to the nonrelativistic effective theory (NREFT) with the quarks (and larger momenta) integrated out. The theory describes dynamics of heavy quirk- antiquirk pairs at energy scales in the center-of-mass frame much smaller than their masses. In Eq. (A6), we have reproduced the NREFT Lagrangian. In quarkonium physics, a higher degree of simplification has been achieved by exploiting $m v \gg m v^{2}$ and building the so-called potential-NREFT (or pNREFT) [58,60], where degrees of freedom of $\sim m v$ are integrated out. In this way, an analytical calculation of the spectrum becomes possible. The ultrasoft degrees of freedom that remain dynamical in this theory are quirks of momentum $m v$ and energy $m v^{2}$ and quirky gauge fields of momentum and energy less than $m v^{2}$. The matching of the pNREFT to the NREFT is perturbative as long as $m v^{2} \gtrsim \Lambda_{Q}$.

The low energy theory is described in terms of quirk bilinears, which depend on the relative distance between the two quirks, $\vec{r} \equiv \vec{x}_{1}-\vec{x}_{2}$, and the center-of-mass coordinate $\vec{R} \equiv\left(\vec{x}_{1}+\vec{x}_{2}\right) / 2$. All gauge fields are multipole expanded in $\vec{r}$, and therefore depend only on $R$. At leading order in the multipole expansion $[59,60]$,

$$
\mathcal{L}=S^{\dagger}\left(i \partial_{t}+\frac{1}{2 \mu} \vec{\partial}_{r}^{2}-V_{s}(r)\right) S .
$$

In the above, $S$ is any quirkcolor singlet field and $V_{s}(r)$ is the matching potential. The reduced mass of the system is denoted by $\mu$. For our model,

$$
\frac{1}{\mu}=\frac{1}{m_{U}}+\frac{1}{m_{D}} .
$$

Hence, at leading order in the multipole expansion, the equation of motion of the singlet field is simply the Schrödinger equation. Determining the bound state energies is thus very similar to a standard quantum mechanical calculation. The main difference is that the potential depends on a scale-dependent quirkcolor coupling that introduces $\log r$ dependence in the potential.

\section{APPENDIX B: UD PARITY}

In this Appendix we demonstrate the charge radius operator is odd under a certain $Z_{2}$ symmetry under which the $U$ quirk transforms to $D$ quirk, which we call "UD parity." Imposing that the ultraviolet theory is UD-parity symmetric therefore eliminates the charge radius operator and automatically ensures the lightest baryon is electrically neutral.

Consider the limit $m_{U}=m_{D}$. In the ultraviolet theory, one can show that the Lagrangian is symmetric under the following transformation:

$$
\begin{gathered}
\xi_{U} \rightarrow \xi_{D} \text { and } \xi_{\bar{U}} \rightarrow \xi_{\bar{D}} \Rightarrow \psi_{U} \rightarrow \psi_{D} \text { and } \chi_{U} \rightarrow \chi_{D} \\
A_{q}^{\mu} \rightarrow A_{q}^{\mu} \text { and } A^{\mu} \rightarrow-A^{\mu} .
\end{gathered}
$$

In the above $A_{q}^{\mu}$ and $A^{\mu}$ are the quirkcolor gauge fields and the electromagnetic gauge fields, respectively. Under UD parity, the electrically neutral scalar baryon is odd and the vector baryon is even, 
QUIRKY COMPOSITE DARK MATTER

$$
B_{0} \rightarrow-B_{0} \quad B^{A I} \rightarrow B^{A I}
$$

and thus

$$
E_{i} \rightarrow-E_{i} \quad B_{i} \rightarrow-B_{i} \quad r_{D}^{2} \rightarrow-r_{D}^{2}
$$

Hence, $r_{D}^{2}$ must vanish if the low energy theory preserves UD parity.

\section{APPENDIX C: INTEGRATION RESULTS}

We have verified the results in [47] including their Appendix, except for the expression for their $c_{g}$ in (A4).
We find the correct expression is

$$
c_{g}=\frac{2 \Delta M^{2}}{M_{*} \Gamma_{*}}+\frac{2 M^{2}}{M_{*} \Gamma_{*}} \frac{\Delta M^{2}-m_{*}^{2} \Gamma_{*}^{2}}{\Delta M^{2}+m_{*}^{2} \Gamma_{*}^{2}},
$$

where $\Delta M^{2} \equiv M_{*}^{2}-M^{2}$. With this correction, we were able to reproduce the numerical results of Ref. [47] (including their Fig. 3).
[1] R. S. Chivukula and T. P. Walker, Nucl. Phys. B329, 445 (1990).

[2] S. M. Barr, R. S. Chivukula, and E. Farhi, Phys. Lett. B 241, 387 (1990).

[3] R.S. Chivukula, A. G. Cohen, M.E. Luke, and M. J. Savage, Phys. Lett. B 298, 380 (1993).

[4] J. Bagnasco, M. Dine, and S. D. Thomas, Phys. Lett. B 320, 99 (1994).

[5] S. B. Gudnason, C. Kouvaris, and F. Sannino, Phys. Rev. D 74, 095008 (2006).

[6] R. Foadi, M. T. Frandsen, and F. Sannino, Phys. Rev. D 80, 037702 (2009).

[7] D. B. Kaplan, Phys. Rev. Lett. 68, 741 (1992).

[8] T. Banks, J. D. Mason, and D. O’Neil, Phys. Rev. D 72, 043530 (2005).

[9] M. Fujii and T. Yanagida, Phys. Lett. B 542, 80 (2002).

[10] D. Hooper, J. March-Russell, and S. M. West, Phys. Lett. B 605, 228 (2005).

[11] R. Kitano and I. Low, Phys. Rev. D 71, 023510 (2005).

[12] N. Cosme, L. Lopez Honorez, and M. H. G. Tytgat, Phys. Rev. D 72, 043505 (2005).

[13] G. R. Farrar and G. Zaharijas, Phys. Rev. Lett. 96, 041302 (2006).

[14] D. Suematsu, J. Cosmol. Astropart. Phys. 01 (2006) 026.

[15] R. Kitano, H. Murayama, and M. Ratz, Phys. Lett. B 669, 145 (2008).

[16] L. Roszkowski and O. Seto, Phys. Rev. Lett. 98, 161304 (2007); O. Seto and M. Yamaguchi, Phys. Rev. D 75, 123506 (2007); M. Aoki, S. Kanemura, and O. Seto, Phys. Rev. D 80, 033007 (2009).

[17] D. E. Kaplan, M. A. Luty, and K. M. Zurek, Phys. Rev. D 79, 115016 (2009).

[18] J. Angle et al. (XENON Collaboration), Phys. Rev. Lett. 100, 021303 (2008).

[19] Z. Ahmed et al. (CDMS Collaboration), Phys. Rev. Lett. 102, 011301 (2009).

[20] J. L. Diaz-Cruz, Phys. Rev. Lett. 100, 221802 (2008).

[21] M. J. Strassler and K. M. Zurek, Phys. Lett. B 651, 374 (2007).

[22] J. Kang and M. A. Luty, arXiv:0805.4642.

[23] J. Preskill, Nucl. Phys. B177, 21 (1981).
[24] M.E. Peskin and T. Takeuchi, Phys. Rev. D 46, 381 (1992).

[25] G. D. Kribs, T. Plehn, M. Spannowsky, and T. M. P. Tait, Phys. Rev. D 76, 075016 (2007).

[26] C. Amsler et al. (Particle Data Group), Phys. Lett. B 667, 1 (2008).

[27] K. Cheung, W. Y. Keung, and T.C. Yuan, Nucl. Phys. B811, 274 (2009).

[28] J. E. Juknevich, D. Melnikov, and M. J. Strassler, J. High Energy Phys. 07 (2009) 055.

[29] W. Fischler, Nucl. Phys. B129, 157 (1977).

[30] A. Billoire, Phys. Lett. 92B, 343 (1980).

[31] N. Brambilla et al. (Quarkonium Working Group), arXiv: hep-ph/0412158.

[32] J. A. Harvey and M. S. Turner, Phys. Rev. D 42, 3344 (1990).

[33] R. Fok and G. D. Kribs, Phys. Rev. D 78, 075023 (2008).

[34] F. R. Klinkhamer and N. S. Manton, Phys. Rev. D 30, 2212 (1984).

[35] A. D. Dolgov, Phys. Rep. 222, 309 (1992).

[36] M. Pospelov and A. Ritz, Phys. Rev. D 78, 055003 (2008).

[37] D. S. M. Alves, S.R. Behbahani, P. Schuster, and J. G. Wacker, arXiv:0903.3945.

[38] D. E. Kaplan, G.Z. Krnjaic, K. R. Rehermann, and C. M. Wells, arXiv:0909.0753.

[39] C.P. Burgess, M. Pospelov, and T. ter Veldhuis, Nucl. Phys. B619, 709 (2001).

[40] S. Andreas, T. Hambye, and M. H. G. Tytgat, J. Cosmol. Astropart. Phys. 10 (2008) 034.

[41] J. R. Ellis, A. Ferstl, and K. A. Olive, Phys. Lett. B 481, 304 (2000).

[42] M. A. Shifman, A. I. Vainshtein, and V. I. Zakharov, Phys. Lett. 78B, 443 (1978).

[43] H. Ohki et al., Phys. Rev. D 78, 054502 (2008).

[44] J. Giedt, A. W. Thomas, and R. D. Young, Phys. Rev. Lett. 103, 201802 (2009).

[45] M. Pospelov and T. ter Veldhuis, Phys. Lett. B 480, 181 (2000).

[46] J. W. Chen and M. J. Savage, Phys. Rev. D 57, 2837 (1998).

[47] S. Profumo and K. Sigurdson, Phys. Rev. D 75, 023521 (2007). 
[48] N. Brambilla, Y. Jia, and A. Vairo, Phys. Rev. D 73, 054005 (2006).

[49] S. Weinberg, The Quantum Theory of Fields. Vol. 1: Foundations (Cambridge University Press, Cambridge, UK, 1995), p. 609.

[50] M. S. Warren, K. Abazajian, D. E. Holz, and L. Teodoro, Astrophys. J. 646, 881 (2006).

[51] A. E. Evrard et al., Astrophys. J. 672, 122 (2008).

[52] S. Schanne (INTEGRAL/SPI Collaboration), J. Phys. Conf. Ser. 41, 46 (2006).

[53] H. Goldberg, G. Perez, and I. Sarcevic, J. High Energy Phys. 11 (2006) 023.

[54] D. Hooper, Phys. Rev. D 75, 123001 (2007).

[55] C. Kilic, T. Okui, and R. Sundrum, J. High Energy Phys. 02 (2010) 018.

[56] R. Harnik and T. Wizansky, Phys. Rev. D 80, 075015 (2009).

[57] W. E. Caswell and G. P. Lepage, Phys. Lett. 167B, 437
(1986).

[58] A. Pineda and J. Soto, Nucl. Phys. Proc. Suppl. 64, 428 (1998).

[59] N. Brambilla, A. Pineda, J. Soto, and A. Vairo, Phys. Rev. D 60, 091502 (1999).

[60] N. Brambilla, A. Pineda, J. Soto, and A. Vairo, Nucl. Phys. B566, 275 (2000).

[61] M. I. Eides, H. Grotch, and V. A. Shelyuto, Phys. Rep. 342, 63 (2001).

[62] S. Recksiegel and Y. Sumino, Phys. Lett. B 578, 369 (2004).

[63] B. A. Kniehl, A. A. Penin, A. Pineda, V. A. Smirnov, and M. Steinhauser, Phys. Rev. Lett. 92, 242001 (2004).

[64] N. Brambilla, A. Pineda, J. Soto, and A. Vairo, Rev. Mod. Phys. 77, 1423 (2005).

[65] S. G. Karshenboim, Phys. Rep. 422, 1 (2005).

[66] E. Eichten, S. Godfrey, H. Mahlke, and J. L. Rosner, Rev. Mod. Phys. 80, 1161 (2008). 